\title{
PRÉ-NATAL DE ALTO RISCO DECORRENTE DE LESÃO MEDULAR: uma análise direcionada a saúde mental
}

DOI: 10.48140/digitaleditora.2021.005.1

Karolayne Brito Silva

Acadêmica de enfermagem do Centro Universitário de Ciências e Tecnologia do Maranhão UniFacema Caxias-MA

(iD) https://orcid.org/0000-

Maria Eduarda Leal de Carvalho Santos

Acadêmica de enfermagem do Centro Universitário de Ciências e Tecnologia do Maranhão UniFacema

Caxias-MA

(iD) https://orcid.org/0000(iD) 0003-3400-0570

Pedro Henrique Medeiros de

\section{Andrade}

Acadêmico de enfermagem do Centro Universitário de Ciências e Tecnologia do Maranhão UniFacema

(iD) https://orcid.org/0000-
Najra Danny Pereira Lima

Mestre em Análise do

Comportamento Aplicada

Centro Paradigma/ Docente do

Centro Universitário de Ciências

e Tecnologia do Maranhão

UniFacema

Caxias-MA

(iD) $0003-3673-0876$

Gilma Sannyelle Silva Rocha

Nutricionista formada pelo Centro Universitário de Ciências e Tecnologia do Maranhão UniFacema e pós graduada em nutrição e pediatria pelo Instituto de Pesquisas Ensino e Gestão em Saúde - IPGS

Caxias-MA

(ID) https://orcid.org/0000-

(iD) 0001-9270-9196

\section{Joyce de Sousa Leal}

Acadêmica de Enfermagem do Centro Universitário de Ciências e Tecnologia do Maranhão UniFacema Caxias-MA

(iD) https://orcid.org/0000-
Simone Costa de Sousa Acadêmica de Enfermagem do Centro Universitário de Ciências e Tecnologia do Maranhão UniFacema Caxias-MA (iD) https://orcid.org/0000-

Rondinelle dos Santos Chaves Enfermeiro Especialista em Preceptoria no SUS (Instituto Sírio Libanês de Ensino e Pesquisa, IEPSL)

São Paulo-SP

(iD) https://orcid.org/0000-

(iD) 0003-4941-8005/print

Mayanny da Silva Lima

Enfermeira Especialista em saúde pública/PSF pelo Instituto Athenas. Caxias-MA

(iD) http://orcid.org/0000 0002-6955-5675

Joerdanyelle Camilla Passos de Oliveira Rabelo

Enfermeira Especialista em Enfermagem do Trabalho- Instituto Athena

Caxias-MA

(iD) https://orcid.org/00000001-7997-9570 


\section{PRÉ-NATAL DE ALTO RISCO DECORRENTE DE LESÃO MEDULAR: uma análise direcionada a saúde mental}

DOI: 10.48140/digitaleditora.2021.005.1

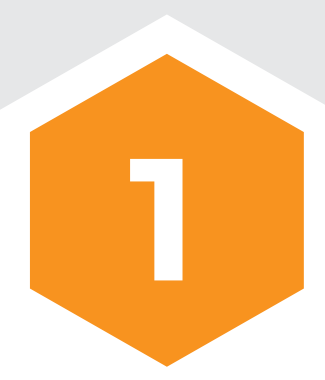

RESUMO

Recebido em: 30/11/2020

Aprovado em: 10/12/2020

Conflito de Interesse: não

Suporte Financeiro: não houve

Objetivos: De forma geral, objetivou-se realizar a consulta de pré-natal a paciente com transtornos mentais; e de forma específica realizar a evolução da paciente; elaborar um plano de cuidados respaldado em North American Nursing Diagnosis Association, NICNursing Interventions Classification, e NOC- Nursing Outcomes Classification; e descrever as atividades realizadas.

Métodos: Trata-se de um relatório de estágio, realizado em uma UBS (unidade básica de saúde) do município de Caxias - MA. Para realização do trabalho foi necessário o período de 22 dias para coleta de dados da paciente, utilizando como fontes: consulta de enfermagem, prontuários, exames realizados e prescrições médicas.

Resultados: Foram realizadas diversas atividades, tanto assistências quanto administrativas, no qual as principais foram acolhimento humanizado e orientações quanto ao atendimento; consulta de enfermagem (saúde mental); consulta de pré-natal; consulta de puericultura e puerpério; realização de ações educativas para promoção da saúde da mulher e do adolescente, entre outras.

Conclusão: O estudo possibilitou expandir os conhecimentos acerca do pré-natal e demais atividades realizadas nas unidades básicas de saúde, além de permitir montar planos de cuidados desenvolvidos com foco nas gestantes que apresentam lesões medulares e os problemas enfrentados por elas. 


\section{HIGH RISK PRENATAL ARISING FROM SPINAL INJURY: an analysis focused on mental health}

DOI: 10.48140/digitaleditora.2021.005.1

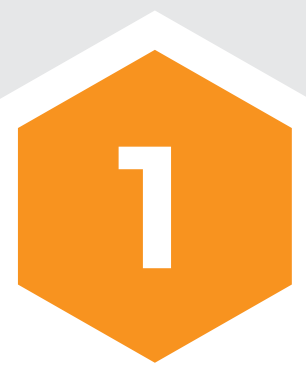

ABSTRACT

Recebido em: 30/11/2020

Aprovado em: 10/12/2020

Conflito de Interesse: não

Suporte Financeiro: não houve

Objectives: In general, it was aimed to realize the prenatal consultation to the patient with mental disorders; and in a specific way to realize the patient's evolution; elaborate a care plan supported by the North American Nursing Diagnosis Association, NIC - Nursing Interventions Classification, and NOC - Nursing Outcomes Classification; and describe the activities performed. the commissions as well as to point out new possibilities of action.

Methods: This is an internship report, realized in a UBS (basic health unit) in the city of Caxias- MA. To accomplish the work it was necessary the period of 22 days to collect data of the patient, using as sources: nursing consultation, medical records, exams carried through and prescriptions.

Results: Several activities were carried through, as much assistance as administrative, in which the main ones were humanized reception and orientation as to the attendance; nursing consultation (mental health); prenatal consultation; childcare and puerperium consultation; accomplishment of educative actions to promote the woman and adolescent's health, among others.

Conclusion: The study made possible to expand the knowledge about the prenatal and other activities carried through in the basic units of health, besides allowing to mount plans of care developed with focus in the pregnant women that present spinal injuries and the problems faced by them. 


\section{INTRODUÇÃO}

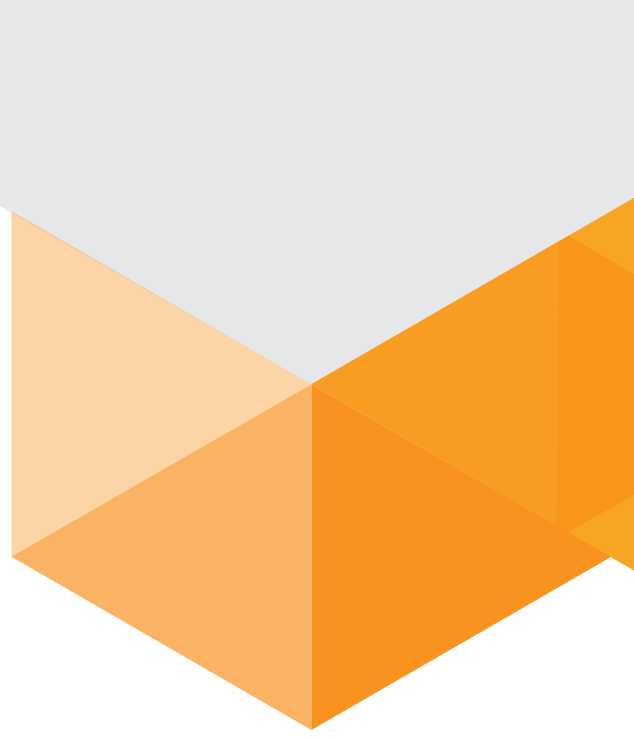

A princípio, a assistência pré-natal inclui um conjunto de ações direcionadas a prevenção de doenças e promoção da saúde da mulher, visando à realização de partos seguros e recém-nascidos saudáveis sem impactos negativos na saúde de ambos. Além disso, a classificação de risco na gestação faz parte da rotina do pré-natal, uma vez que, o histórico familiar, antecedentes gestacional/obstétrico integram a consulta da gestante. Nesse sentido, as gestações de alto risco requerem monitoramento rigoroso e, às vezes, encaminhamento para o CEAMI (centro especializado ambulatorial materno infantil) (MENDES et al., 2020).

Os autores Silva et al. (2010) acrescentam que a gestação é um período de modificações fisiológicas e hormonais que podem influenciar no seu bem estar físico, psicológico e social, em vista disso, pode ocorrer a não aceitação da gestação e consequentemente ocasionar alterações psicológicas, podendo assim, ser um período em que verifica o aumento das sintomatologias de doenças preexistentes, ou até mesmo, o desenvolvimento de transtornos psiquiátricos. Em vista disso, os transtornos mentais mais comuns incluem sintomas depressivos não psicóticos, ansiedade e queixas somáticas que afetam o desempenho das atividades diárias.

Apesar da lesão medular não está relacionada a nenhum tipo de alteração hormonal ou ginecológica, e não haver evidências científicas que comprovam a impossibilidade de gravidez. A mesma pode causar ricos graves na gestação, uma vez que, uma lesão medular envolve sérias consequências físicas, sociais e psicológicas. As respectivas sequelas não só limitam e causam dependência como também exige um longo processo de reabilitação. Então consequentemente, a gestante tende a desenvolver transtornos psicológicos como ansiedade e depressão, visto que, as alterações fisiológicas associadas à gravidez poderão ser agravadas em decorrência da lesão medular (MATIAS; SANTOS; CERQUEIRA, 2014).

Segundo Silva et al. (2019) os transtornos psicológicos têm manifestações variadas, comumente, são caracterizados pela associação de pensamentos, emoções e comportamento anormais, que podem afetar as relações sociais, uma vez que, os sintomas afetam o indivíduo em suas funções cognitivas, físicas, emocionais e comportamentais, com influência negativa em suas atividades cotidianas. Entre os transtornos mentais, estão à depressão, ansiedade, transtorno afetivo bipolar, e outras psicoses. 
Diante do exposto, apresenta-se a necessidade de avaliar a assistência de enfermagem no pré-natal de paciente com lesão medular com foco na saúde mental, para tal objetivou-se de forma geral realizar a consulta de pré-natal a paciente com transtornos mentais; e de forma específica realizar a evolução da paciente; elaborar um plano de cuidados respaldado em North American Nursing Diagnosis Association (NANDA, 2018), NIC- Nursing Interventions Classification (MCCLOSKEY; BULECHEK, 2008), e NOC- Nursing Outcomes Classification (MOORHEAD, 2010); e descrever as atividades realizadas.

\section{DESENVOLVIMENTO}

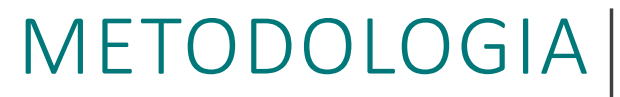

Trata-se de um relatório de estágio, realizado em uma UBS (unidade básica de saúde) do município de Caxias - MA. Para realização do trabalho foi necessário o período de 22 dias para coleta de dados da paciente, utilizando como fontes: consulta de enfermagem, prontuários, exames realizados e prescrições médicas.

\section{HISTÓRICO DE ENFERMAGEM}

\section{Caso Clínico- 05/10/2020 ás 09: 30hrs}

\section{Anamnese:}

Paciente B. C. D, 36 anos de idade, sexo feminino, alfabetizada, solteira, cor parda, multigesta, primeiro parto vaginal com histórico pessoal de ansiedade, depressão e lesão medular. Histórico familiar de gemelares e hipertensão na família. Comparece a UBS para iniciar o pré-natal com ultrassonografia obstétrica realizada em 01/10/2020, com idade gestacional de 10 semanas e 4 dias, com a DUM 18/07/2020, DPP (dum) 24/04/2021, DPP (eco) 29/04/2021. Queixa-se de azia, cefaleia e fraqueza.

Apresenta lesão medular da coluna cervical: espondilose cervical, abaulamento posterior difuso do anel discal C4, C5, abaulamento posterior difuso no nível C5, C6, C1 com protrusão discal. RM da coluna lombar: sinais de manipulação cirúrgica no nível L5- S1 e laminectomia em L5 á esquerda: espondilodiscopatia degenerativa lombar. Pequena hérnia discal extrusa posterior paracentral esquerda no nível L5 - S. Refere ter realizado cirurgia de hernia no dia 13/11/2019. Em uso de amytril + carbamazepino, mantido pelo psiquiatra segundo informação obtida. Encaminhada ao CEAMI (centro especializado ambulatorial materno infantil) por apresentar vários problemas de saúde. Aguardo exames 10 trimestre; testes rápidos $\mathrm{HBsAg}, \mathrm{HCV}$, Siflis e HIV. Encaminhada a sala odontológica. Em uso de sulfato ferroso e ácido fólico. Segue orientação geral. Aguardo retorno médico. 


\section{Exame Físico (céfalo-caudal):}

Paciente encontra-se lúcida, orientada em tempo e espaço, bom estado geral, normotensa, eupneica, normoesfigmo, afebril, hidratada, mucosas normocoradas, perfusão capilar normal, saturação satisfatória. Pele íntegra, normocorada, normotérmica, textura de aspecto flácido, sem alterações, tugor firme, estrias presentes; cabelos não apresentam seborréia ou pediculosidade; couro cabeludo íntegro sem anormalidades, lesões ou nódulos; unhas não apresentam infeções fúngicas, lúnula presente, sem alterações. Crânio normocefálico, sem abaulamentos ou ondulações. Face assimétrica, normocorada, ausência de cloasma, pouca distribuição de pelos. Globo ocular sem alterações (blefaroptose ou ptose palpebral, exoftalmia/protrusão, microftalmia, hipertelorismo, nistagmo, entre outras), pupilas isocóricas, fotorreagentes, paciente refere miopia. Nariz com higiene satisfatória sem sujidades, não apresenta desvio de septo, olfato presente, sem alterações (obstrução nasal, rinorreia, pertubações reflexas, epistaxe). Lábios simétricos, rosados, íntegros, lisos, úmidos, sorriso simétrico, não apresenta halitose, dentição completa, dentes lisos, amarelados e brilhantes, língua de textura lisa, uniforme, sem lesões, rosada, com veias entre o frênulo e as dobras, palato duro forma de cúpula de coloração esbranquiçada, palato mole sem alterações, mobilidade do palato e da úvula presentes, orelhas sem alterações visíveis (otalgia, otorréia, tinitus), pavilhão auricular sem alterações (manchas, escamações, hipervascularização, nódulos, depressões). Pescoço com mobilidade satisfatória, sem nódulos, linfonodos palpáveis indolor, glândula tireóide palpável, mobilidade ao deglutir á seco presente, carótida e jugular sem alterações. MMSS sem alterações (edemas, hematomas, manchas eritematosas). Avaliado cruzamento da linha hemiclavicular esquerda com o 5o espaço intercostal. Inspeção estática: ausência de lordose, cifose ou escoliose, sem alterações estruturais como pectus excavatum, ou o tórax em barril. Inspeção dinâmica: eupneico, expansibilidade simétrica, frêmito torácico vocal (FTV) sem alterações, na percussão, som claro pulmonar, abaixo do 6o espaço intercostal direito é encontrado um som maciço correspondendo ao fígado (percussão executada na porção anterior do tórax). Na ausculta, murmúrios vesiculares presentes. Mamas aumentadas de volume em consequência da hipertrofia, auréola escura, mamilos protusos, ausência de colostro. A.C: precórdio calmo, sem abaulamentos, retrações ou deformidades, ictus cordis palpável 1-2 polpas digitais na linha hemiclavicular esquerda no 5을.C. Bulhas cardíacas (B1 e B2) rítmicas, normofonéticas em dois tempos, sem sopros. AD: Abdômen globoso ou ovóide, cicatriz umbilical plana, hiperpigmentação da linha alva (linea nigra). No aparelho genital externo alterações como mucosa hiperpigimentada, tumufeita, cianosada, mais intensificado no vestíbulo e nas proximidades do meato urinário. Mensuração: AU ausente; I.G (eco): 10 semanas e 4 dias; I.G (dum): 11 semanas e 2 dias; movimentos fetais ausentes, bcf ausentes. MMIl: sem alterações (edemas, hematomas, manchas eritematosas, dilatação circunscrita de vasos sanguíneos). Sono e repouso insatisfatório. SSVV- PA: 110x60mmhg; FC: 20 irpm; SO2 99\%; Peso: 64,5kg; Altura: 1,56; IMC: 26,5. 


\section{EVOLUÇÃO DE ENFERMAGEM}

(Evolução do segundo dia de coleta) Data da consulta: 07/12/2020 ás 10: 00hrs

Paciente B. C. D, 36 anos de idade, sexo feminino, alfabetizada, solteira, cor parda, multigesta, primeiro parto vaginal com histórico pessoal de ansiedade, depressão e lesão medular. Histórico familiar de gemelares e hipertensão na família. Comparece a UBS para consulta de pré-natal com a DUM 18/07/2020, DPP (dum) 24/04/2021, DPP (eco): 29/04/2021. Queixando-se de azia, lombalgia e "dor na região da cirurgia".

Exame Físico (céfalo-caudal):

Paciente encontra-se lúcida, orientada em tempo e espaço, bom estado geral, normotensa, eupneica, normoesfigmo, afebril, hidratada, mucosas normocoradas, perfusão capilar normal, saturação satisfatório. Pele íntegra, normocorada, normotérmica, textura de aspecto flácido, sem alterações, tugor firme, estrias presentes; cabelos não apresentam seborréia ou pediculosidade; couro cabeludo íntegro sem anormalidades, lesões ou nódulos; unhas não apresentam infeções fúngicas, lúnula presente, sem alterações. Crânio normocefálico, sem abaulamentos ou ondulações. Face assimétrica, normocorada, ausência de cloasma, pouca distribuição de pelos na face. Globo ocular sem alterações (blefaroptose ou ptose palpebral, exoftalmia/protrusão, microftalmia, hipertelorismo, nistagmo, entre outras), pupilas isocóricas, fotorreagentes, paciente refere miopia. Nariz com higiene satisfatória sem sujidades, não apresenta desvio de septo, olfato presente, sem alterações (obstrução nasal, rinorreia, pertubações reflexas, epistaxe). Boca com lábios simétricos, rosados, íntegros, lisos, úmidos, sorriso simétrico, não apresenta halitose, dentição completa, dentes lisos, amarelados e brilhantes, língua de textura lisa, uniforme, sem lesões, rosada, com veias entre o frênulo e as dobras, palato duro forma de cúpula de coloração esbranquiçada, palato mole sem alterações, mobilidade do palato e da úvula presentes, orelhas sem alterações visíveis (otalgia, otorréia, tinitus), pavilhão auricular sem alterações (manchas, escamações, hipervascularização, nódulos, depressões). Pescoço com mobilidade satisfatória, sem nódulos, linfonodos palpáveis indolor, glândula tireóide palpável, mobilidade ao deglutir á seco presente, carótida e jugular sem alterações. MMSS sem alterações (edemas, hematomas, manchas eritematosas). Avaliado cruzamento da linha hemiclavicular esquerda com o 50 espaço intercostal. Inspeção estática: ausência de lordose, cifose ou escoliose, sem alterações estruturais como pectus excavatum ou o tórax em barril. Inspeção dinâmica: eupneico, expansibilidade simétrica, frêmito torácico vocal (FTV) sem alterações, na percussão som claro pulmonar, abaixo do 6o espaço intercostal direito é encontrado um som maciço correspondendo ao fígado (percussão executada na porção anterior do tórax). Na ausculta, murmúrios vesiculares presentes. Mamas aumentadas de volume em consequência da hipertrofia, auréola escura, mamilos protusos, ausência de colostro. A.C: precórdio calmo, sem abaulamentos, retrações ou deformidades, ictus cordis palpável 1-2 polpas digitais na linha hemiclavicular esquerda no 5 o EIC. Bulhas cardíacas (B1 e B2) rítmicas, normofonéticas em dois tempos, sem sopros. AD: abdômen globoso ou ovóide, cicatriz umbilical plana, hiperpigmentação da linha alva (linea nigra). Aparelho genital externo com alterações como mucosa hiperpigimentada, tumufeita, cianosada, mais intensificado no vestíbulo e nas proximidades do meato urinário. Mensuração: AU 14 cm; I.G: 19 semanas e 2 dias pela eco; movimentos fetais ausentes. Palpação: embrião presente, com bcf 170bpm. Exploração do fundo uterino: pólo pélvico, com a característica de ser mais volumoso que a cabeça, de superfície irregular. Exploração do dorso fetal: a região dorsal do feto apresenta-se como superfície resistente e contínua. Exploração da mobilidade: pólo alto e móvel balança de um lado para o outro. Exploração da escava: pólo cefálico ausente. MMII sem alterações (edemas, hematomas, manchas eritematosas, dilatação circunscrita de vasos sanguíneos). Sono e repouso insatisfatório. SSVV- PA: 90x60mmhg; FC: 20 irpm; SO2 98\%; Peso: 65 kg; Altura: 1,56 IMC: 26.71.

Condutas: em uso de amytril $25 \mathrm{mg}$ + sertralina $50 \mathrm{mg}$ + zolpidem10mg; prescrito materno (suplemento vitamínico mineral); encaminhada para sala de vacinação para avaliação da mesma; encaminhada a sala odontológica; conduta orientação geral; agendado retorno. 


\section{DIAGNÓSTICOS DE ENFERMAGEM}

Quadro 1. Distribuição dos diagnósticos de enfermagem identificados no caso clínico de acordo com a taxonomia da NANDA (North American Nursing Diagnosis Association). Caxias-MA. 2020.

\begin{tabular}{|c|c|c|c|c|}
\hline DIAGNÓSTICOS & DOMÍNIO & CLASSE & $\begin{array}{c}\text { CARACTERÍSTICAS } \\
\text { DEFINIDORAS }\end{array}$ & $\begin{array}{c}\text { FATORES } \\
\text { RELACIONADOS }\end{array}$ \\
\hline Ansiedade & $\begin{array}{c}\text { Domínio } 9 \\
\text { Enfrentamen- } \\
\text { to/tolerância } \\
\text { ao estresse. }\end{array}$ & $\begin{array}{c}\text { Classe } 2 \\
\text { Respostas de } \\
\text { enfrentamen- } \\
\text { to. } \\
\text { Código do } \\
\text { diagnóstico } \\
00146 .\end{array}$ & $\begin{array}{l}\text { Comportamentais: Inquieta- } \\
\text { ção, Insônia, Preocupações } \\
\text { em razão de mudança em } \\
\text { eventos da vida, Produtividade } \\
\text { diminuída. } \\
\text { Afetivas: agonia, apreensão, } \\
\text { arrependimento, atos bruscos } \\
\text { desamparo, medo. } \\
\text { Fisiológicas: aumento da ten- } \\
\text { são aumento da transpiração } \\
\text { estremecimentos, tensão facial } \\
\text { tremores. } \\
\text { Simpática: Alteração no padrão } \\
\text { respiratório, Anorexia Aumento } \\
\text { da frequência cardíaca, Au- } \\
\text { mento da frequência respirató- } \\
\text { ria, Aumento da pressão arte- } \\
\text { rial, Boca seca. } \\
\text { Parassimpáticas: Alteração no } \\
\text { padrão de sono, diminuição da } \\
\text { pressão arterial, urgência uri- } \\
\text { nária. } \\
\text { Cognitivas: Alteração na con- } \\
\text { centração, bloqueio de pensa- } \\
\text { mentos, campo de percepção } \\
\text { diminuído, capacidade diminuí- } \\
\text { da para aprender, capacidade } \\
\text { diminuída para solucionar pro- } \\
\text { blemas. }\end{array}$ & $\begin{array}{l}\text { Conflito de valores; } \\
\text { Conflito sobre as metas } \\
\text { da vida; } \\
\text { Contágio interpessoal; } \\
\text { Estressores; Necessi- } \\
\text { dades não atendidas; } \\
\text { Transmissão interpes- } \\
\text { soal. }\end{array}$ \\
\hline Insônia & $\begin{array}{c}\text { Domínio } 4 \\
\text { Atividade/re- } \\
\text { pouso. }\end{array}$ & $\begin{array}{c}\text { Classe } 1 \\
\text { Sono/repou- } \\
\text { so. } \\
\text { Código do } \\
\text { diagnóstico } \\
00095 .\end{array}$ & $\begin{array}{l}\text { Alteração na concentração, al- } \\
\text { teração no afeto, alteração no } \\
\text { humor, alteração no padrão de } \\
\text { sono, aumento de acidentes, } \\
\text { dificuldade para iniciar o sono, } \\
\text { dificuldade para manter o sono, } \\
\text { energia insuficiente, estado de } \\
\text { saúde comprometido, redução } \\
\text { na qualidade de vida. }\end{array}$ & $\begin{array}{l}\text { Ansiedade, depressão, } \\
\text { desconforto físico, es- } \\
\text { tressores, média de } \\
\text { atividade física diária } \\
\text { inferior à recomenda- } \\
\text { da para idade e sexo, } \\
\text { medo. } \\
\text { Obs. Condições asso- } \\
\text { ciadas a Agentes far- } \\
\text { macêuticos e Alteração } \\
\text { hormonal. }\end{array}$ \\
\hline
\end{tabular}


Classe 3

Conforto social

Social

\section{ineficaz da saúde}

Manutenção

\section{Domínio 1}

Promoção da saúde.

Domínio 11

Queda

$$
\begin{aligned}
& \text { Segurança/ } \\
& \text { proteção }
\end{aligned}
$$

\section{Síndrome da dor crônica}

Sofrimento espiritual

\section{Domínio 12 \\ Conforto}

\section{Sobrepeso}

Domínio 2

Nutrição

\section{Domínio 10}

Princípios da vida
Código do

diagnóstico 00053.

Classe 2

\section{Controle da} saúde

Código do diagnóstico 00099.

Classe 2

Lesão física

Código do diagnóstico 00155.

Classe 1

\section{Conforto físico}

Código do diagnóstico 00255.

\section{Classe 1}

Ingestão

Código do diagnóstico

ADULTO: Índice de massa corporal $($ IMC) $>25 \mathrm{~kg} / \mathrm{m} 2$ 00233.

\section{Classe 3}

Coerência entre valores/ crenças/atos Código do diagnóstico 00066 . próprios pensamentos, retraimento.

Apoio social insuficiente, incapacidade de assumir a responsabilidade de atender a práticas básicas de saúde, padrão de ausência de comportamento de busca de saúde.

Condições associadas a agente farmacêutico, alteração na função cognitiva, hipotensão ortostática.

Ansiedade, distúrbio no padrão social, sobrecarga de estresse.

Ansiedade, fadiga, insônia medo, questionamento do sentido da vida, questionamento do sentido do sofrimento.

Conexão pessoais: aceitação inadequada, coragem insuficiente, estratégias de enfrentamento ineficazes, falta percebi-
Afeto superficial, ausência de propósito, ausência de sistema de apoio, doença, história de rejeição, preocupação com os de sono, insônia, isolamento A serem desenvolvidos. da de sentido na vida.
Recursos pessoais insuficientes.

Condições associadas, alteração na aparência física, alteração no bem-estar, alteração no estado mental.

Estratégias de enfrentamento ineficazes, recursos insuficientes, sofrimento espiritual, tomada de decisões prejudicada.

Fatores de risco: Ausência de sono, desmaio ao estender o pescoço, desmaio ao virar o pescoço.

Conhecimento insuficiente sobre os fatores modificáveis, distúrbio do sono, pouco gasto de energia,

Ansiedade, baixa autoestima, depressão, dor, estressores.

Fonte: Adaptado de Nanda (2018). 


\section{RESULTADOS ESPERADOS}

Quadro 2. Classificação dos resultados esperados de enfermagem de acordo com NOC (Nursing Outcomes Classification). Caxias-MA. 2020.

\begin{tabular}{|c|c|c|}
\hline DIAGNÓSTICOS & RESULTADOS SUGERIDOS & RESULTADOS ADICIONAIS ASSOCIADOS \\
\hline Ansiedade & $\begin{array}{l}\text { Autocontrole da ansiedade, con- } \\
\text { centração, enfrentamento do nível } \\
\text { de ansiedade e de nível de hipera- } \\
\text { tividade. }\end{array}$ & $\begin{array}{l}\text { Aceitação do estado de Saúde; } \\
\text { Ajuste Psicossocial relacionado a mudança de } \\
\text { estilo de Vida; } \\
\text { Controle de Sintomas; } \\
\text { Desenvolvimento de habilidades de Interação } \\
\text { Social. }\end{array}$ \\
\hline Insônia & $\begin{array}{l}\text { Bem-estar Pessoal; Concentração; } \\
\text { Equilíbrio do Humor, e estado de } \\
\text { Saúde Pessoal; Nível de Fadiga; } \\
\text { Qualidade de Vida e Repouso. }\end{array}$ & $\begin{array}{l}\text { Nível de Ansiedade; Nível de Depressão; Nível } \\
\text { de Desconforto; Nível de Dor; Nível de Estresse; } \\
\text { Nível de Medo e Resposta à Medicação. }\end{array}$ \\
\hline $\begin{array}{l}\text { Manutenção ine- } \\
\text { ficaz da saúde }\end{array}$ & $\begin{array}{l}\text { Autocuidado: Alimentação, realiza- } \\
\text { ção de Atividades da Vida Diária } \\
\text { (AVD), Atividades Instrumentais da } \\
\text { Vida Diária (AIVD), Higiene, Auto- } \\
\text { nomia Pessoal; Comportamento } \\
\text { de Aceitação: Dieta Prescrita; Me- } \\
\text { dicação Prescrita. }\end{array}$ & $\begin{array}{l}\text { Autodireção dos Cuidados Bem-estar Pessoal; } \\
\text { Comportamento de Adesão: Dieta Saudável; } \\
\text { Comportamento de Busca da Saúde. }\end{array}$ \\
\hline Queda & $\begin{array}{l}\text { Ambiente Domiciliar Seguro; Auto- } \\
\text { cuidado: Uso do Banheiro; Com- } \\
\text { portamento de Compensação da } \\
\text { Visão; Comportamento de Preven- } \\
\text { ção de Quedas; Conhecimento: } \\
\text { Prevenção de Quedas; Controle } \\
\text { de Riscos; Deslocamento Seguro; } \\
\text { Detecção de Riscos; Nível de Agi- } \\
\text { tação; Nível de Confusão Aguda; } \\
\text { Nível de Dor; Nível de Fadiga; }\end{array}$ & Resposta à Medicação. \\
\hline $\begin{array}{l}\text { Síndrome de } \\
\text { dor crônica }\end{array}$ & Controle da Dor. & $\begin{array}{l}\text { Autocontrole da Depressão; Bem-estar Pessoal; } \\
\text { Conhecimento: Controle da Dor, Sintomas, Nível } \\
\text { de Estresse, Nível de Fadiga. } \\
\text { Qualidade de Vida; Vontade de Viver; } \\
\text { Repouso Satisfação do Cliente: Controle da Dor. }\end{array}$ \\
\hline Sobrepeso & $\begin{array}{l}\text { Autocuidado: Alimentação; } \\
\text { Autocuidado: realização de ativi- } \\
\text { dades da Vida Diária (AVD); } \\
\text { Estado da Deglutição; } \\
\text { Estado Nutricional: Ingestão de } \\
\text { Alimentos e Líquidos. }\end{array}$ & $\begin{array}{l}\text { Autocontrole da Ansiedade; Controle da Dor, Ní- } \\
\text { vel de Agitação; } \\
\text { Resistência; } \\
\text { Satisfação do Cliente: Assistência Funcional. } \\
\text { Satisfação do Cliente: Cuidados Físicos. }\end{array}$ \\
\hline
\end{tabular}


Ajuste Psicossocial relacionado a mudança de estilo de Vida; Autoestima; Autonomia Pessoal;

Sofrimento espiritual

Bem-estar Pessoal; Controle do nível de estresse, ansiedade e depressão; Qualidade de vida e Resiliência pessoal; Estratégias de enfrentamento e habilidades de interação social.
Ajuste Psicossocial: Mudança de Vida; Autocontenção ao Suicídio; Autonomia Pessoal Bem-estar Pessoal; Controle da Dor;

Fonte: Adaptado de Moorhead et al. (2010).

\section{INTERVENÇÕES DE ENFERMAGEM}

Quadro 03. Classificação das intervenções de enfermagem de acordo com NIC (Nursing Interventions

Classification). Caxias-MA. 2020.

\section{INTERVENÇÕES DE ENFERMAGEM}

Apoio Emocional; Ensino: medicamentos prescritos; Ensino: processo da doença; Identificação de Risco; Melhora da educação em saúde; Modificação do Comportamento;

Ansiedade

Insônia

\section{Isolamento social}

Manutenção ineficaz da saúde

Queda

Síndrome de dor crônica

Sobrepeso

Sofrimento espiritual Monitoração de Sinais Vitais; Reconciliação de Medicamentos; Monitoração de políticas de saúde; Prevenção do uso de drogas; Prevenção do suicídio; primeiros socorros; Promoção da integridade familiar; Promoção de esperança; Aromaterapia; Administração de Medicamentos.

Fototerapia: regulação do humor/sono; Melhora do enfrentamento; Administração de medicamentos.

Apoio emocional; Melhora do enfrentamento; Promoção da integridade familiar; Promoção de esperança

Melhora da educação em saúde; Modificação do comportamento; Monitoração de sinais vitais; Assistência no autocuidado: atividades essenciais da vida diária; Consulta.

Monitoração de sinais vitais; Prevenção de quedas, Controle do ambiente: segurança; identificação de Risco; Monitoração dos sinais vitais; orientação antecipada.

Consulta; Administração de analgésicos; Administração de medicamentos; Apoio à tomada de decisão; Controle do ambiente/conforto; Gerenciamento de caso; Controle da dor; Controle de energia; Controle de infecção; Estabelecimento de metas mútuas; Assistência em Exames; Massagem; Musicoterapia; Relaxamento muscular progressivo; Supervisão; Apoio Espiritual; Apoio ao Médico; Terapia de Relaxamento; Toque Terapêutico; Troca de Informações sobre Cuidados de Saúde.

Apoio emocional; Aconselhamento nutricional; Educação em saúde; Encaminhamento; ensino: dieta prescrita; Ensino: indivíduo; Ensino: medicamentos prescritos; Estabelecimento de limites; Promoção do exercício

Apoio emocional; Promoção de esperança; Apoio emocional; Melhora do enfrentamento; Promoção integridade familiar; Apoio espiritual; Apoio familiar; Prevenção do suicídio.

Fonte: Adaptado de Mccloskey e Bulechek (2008). 


\section{DESCRIÇÃO DAS ATIVIDADES REALIZADAS}

As atividades realizadas ao longo do estágio foram das mais variadas, além de combinar conhecimento teórico e prático, foi possível desenvolver habilidades para realizar uma assistência de enfermagem humanizada, acolhedora e eficaz, desenvolvendo relacionamento interpessoal com os pacientes, familiares e equipe de saúde, mesmo sendo difícil efetuar assistência aos pacientes mais fragilizados psicologicamente, fisiologicamente e financeiramente.

Além disso, foi um grande aprendizado observar a superação de obstáculos, onde uma equipe unida com o foco no paciente consegue superar qualquer percalço, reorganizando escalas, cobrindo atividades de colegas e buscando apoio uns nos outros.

Em todas as áreas da UBS foram realizadas diversas atividades, tanto assistências quanto administrativas, no qual as principais foram acolhimento humanizado e orientações quanto ao atendimento; orientações aos pacientes e acompanhantes; triagem de pacientes com verificação de PA, peso, altura, glicemia e classificação de risco; admissão de pacientes, realização de anamnese, exame físico e evolução desde o recém-nascido ao idoso; consulta de enfermagem (saúde mental); consulta de pré- natal, e solicitação de exames do 1 으 trimestre de gestação; consulta de puericultura e puerpério; realização de ações educativas para promoção da saúde da mulher e do adolescente; visita domiciliar para consulta de pré-natal de alto risco, entre outras. 


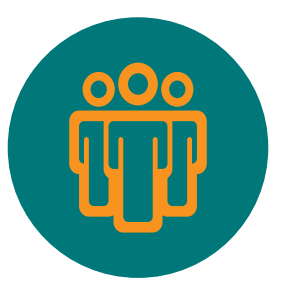

\section{CONCLUSÃO}

Este estudo permitiu observar as consultas de pré-natal realizadas em uma UBS e a realização da prestação da assistência de enfermagem no pré-natal de paciente com lesão medular, dando ênfase à saúde mental das clientes atendidas. Com base no perfil da gestante, foram elaborados diagnósticos de enfermagem e montados planos de cuidado, foi possível ainda, acompanhar as demais atividades realizadas na rotina de uma UBS.

Diante das atividades realizadas, foi possível conhecer mais sobre a dinâmica de funcionamento da UBS e todos os tipos de atendimentos realizados, foi possível acompanhar todas as etapas do pré-natal e evidenciar as peculiaridades no atendimento de gestantes com lesão medular.

Como limitações do estudo tem-se o fato de que seria necessário um maior tempo de estágio para melhor compreender todas as atribuições dos profissionais que atuam na atenção primária, dessa forma, seria possível colocar em prática todos os conhecimentos obtidos pelos estudantes no decorrer da graduação.

Por fim, o estudo possibilitou expandir os conhecimentos acerca do pré-natal e demais atividades realizadas nas unidades básicas de saúde, além de permitir montar planos de cuidados desenvolvidos com foco nas gestantes que apresentam lesões medulares e os problemas enfrentados por elas. Mais estudos sobre a saúde mental de gestantes com lesões medulares devem ser realizados a fim de comprovar a importância de cuidados multidisciplinares as mesmas e os benéficos de intervenções que priorizem a saúde e bem estar da mãe e bebê. 


\section{REFERÊNCIAS}

NANDA INTERNATIONAL. Diagnósticos de enfermagem da NANDA-I: definições e classificação 20182020. 11. ed. Porto Alegre: Artmed, 2018.

MATIAS, A. C.; SANTOS, J. M.; CERQUEIRA, M. E. Gravidez em Lesionadas Medulares: Riscos, Prevenção e Complicações. Revista da Sociedade Portuguesa de Medicina Física e de Reabilitação, [S.I.], v. 26, n. 2, p. 27-35, 2014. DOI: http://dx.doi.org/10.25759/spmfr.145.

MCCLOSKEY, J. C.; BULECHEK, G. M. Classificação das Intervenções de Enfermagem (NIC). 4ạ ed. Porto Alegre: Artmed; 2008.

MENDES, R. B. et al. Avaliação da qualidade do pré-natal a partir das recomendações do Programa de Humanização no Pré-natal e Nascimento. Ciênc. saúde coletiva, Rio de Janeiro, v. 25, n. 3, p. 793-804, mar. 2020. DOI: https://doi.org/10.1590/1413-81232020253.13182018.

MOORHEAD, S. et al. Classificação dos Resultados de Enfermagem (NOC). 2a ed. Porto Alegre: Artmed, 2010.

SILVA, A. C. et al. Prevalência e fatores associados ao transtorno mental comum em assentados rurais*. SMAD, Rev. Eletrônica Saúde Mental Álcool Drog. (Ed. port.), Ribeirão Preto, v. 15, n. 1, p. 23 31, 2019. DOI: http://dx.doi.org/10.11606/issn.1806-6976.smad.2019.000379.

SILVA, R. A. et al. Transtornos mentais comuns e auto-estima na gestação: prevalência e fatores associados. Cad. Saúde Pública, Rio de Janeiro, v. 26, n. 9, p. 1832-1838, set. 2010. DOI: http://dx.doi. org/10.1590/S0102-311X2010000900016. 